\title{
Sarcoma sinovial de extremidad inferior
}

\author{
Lower extremity synovial sarcoma
}

\author{
Francisco José Flores Palomar,* María José Raphael Garza, ${ }^{\ddagger}$ José Jesús Castillo García, ${ }^{\$}$ \\ Guillermo León Merino, ${ }^{\S}$ Claudio René Montes de Oca Orellanall
}

\section{Resumen}

El sarcoma sinovial es una neoplasia mesénquima maligna. Su presentación típica es una masa yuxtaarticular, palpable e indolora. Presentamos el caso de un hombre de 63 años con un tumor en la región inguinal izquierda, en quien se realizó una biopsia escisional, con resultado de hidradenocarcinoma con ganglio regional positivo. Se efectuó inmunohistoquímica con resultado de sarcoma sinovial poco diferenciado. El sarcoma sinovial es una neoplasia de tejidos blandos poco frecuente, que afecta a adultos jóvenes. Se localiza con mayor frecuencia en las extremidades y es común la presencia de metástasis al diagnóstico. Tiene mal pronóstico y el tratamiento es con resección quirúrgica y quimioterapia.

Palabras clave: Sarcoma sinovial, tumor partes blandas, extremidad inferior.

\section{INTRODUCCIÓN}

El sarcoma sinovial es una neoplasia mesenquimal maligna, que con mayor frecuencia se desarrolla adyacente a las articulaciones de las extremidades. ${ }^{1}$ Éste representa entre 5 y $10 \%$ de todos los sarcomas de tejidos blandos, y es el cuarto en frecuencia, después del histiocitoma fibroso maligno, el liposarcoma y el rabdomiosarcoma. ${ }^{2}$ Es más común en adultos jóvenes y no tiene predilección de género, aunque en algunos

\footnotetext{
* Residente de Cirugía General.

* Médico General.

$\S$ Cirujano General.

॥ Cirujano Oncólogo.
}

Hospital Ángeles Pedregal, Ciudad de México, México.

Correspondencia:

Dr. Francisco José Flores Palomar

Correo electrónico: francisco_floresp@hotmail.com

Aceptado: 09-09-2019.

www.medigraphic.com/actamedica

\section{Abstract}

Synovial sarcoma is a malignant mesenchymal neoplasm. Its typical presentation is a juxtaarticular mass, palpable and painless. We present a 63 years old male, with a tumor in the left inguinal region, an excisional biopsy was performed, resulting in a hydradenocarcinoma with a positive regional ganglion. Immunohistochemistry was performed resulting in poorly differentiated synovial sarcoma. Synovial sarcoma is a rare soft tissue neoplasm affecting young adults. It is located more frequently in the extremities and the presence of metastasis at diagnosis is common. It has a poor prognosis and the treatment is with surgical resection and chemotherapy.

Keywords: Synovial Sarcoma, soft-tissue tumor, inferior limb.

estudios se ha reportado que presenta ligera preferencia por el género masculino. ${ }^{3}$

El estudio de Mitsuhashi y su grupo refiere que éste deriva de las células de tejidos blandos más que del tejido sinovial; por ello, es poco común su presentación intraarticular, aunque puede tener origen en cualquier parte del cuerpo. ${ }^{4}$ El origen fuera de las extremidades corresponde al $20 \%$ de los casos y éste puede localizarse en la cabeza y el cuello, el mediastino, el corazón, los pulmones, la pared abdominal, el mesenterio, el retroperitoneo y la cavidad peritoneal. ${ }^{5}$ Otras localizaciones menos frecuentes son el riñón, la próstata y los órganos reproductores femeninos. ${ }^{6}$

Su forma de presentación es de una masa yuxtaarticular, palpable e indolora o poco dolorosa, sin alteraciones funcionales graves. Generalmente son menores de $5 \mathrm{~cm}$, y la mayoría se presenta con metástasis al momento del diagnóstico. ${ }^{7}$

\section{CASO CLÍNICO}

Se trata de un hombre de 63 años, quien acudió a la consulta externa por presentar un tumor en ingle izquierda de $4 \times 4 \mathrm{~cm}$, de consistencia blanda, bien definido, palpable en tejido adiposo del muslo izquierdo. Se decidió realizar 
una biopsia excisional de la lesión y piel circundante, con extensión hasta tejido celular subcutáneo.

En patología, se reportó una lesión de $4.3 \times 3 \mathrm{~cm}$, blanda, bilobulada, con zonas de aspecto hemorrágico, alternada con zonas de tejido amarillo friable y café claro indurada. Se describió como una probable hidradenocarcinoma con infiltración hacia el ganglio linfático (Figuras 1 y 2), con actividad mitósica alta e infiltración local a un ganglio linfático. Se realizó inmunohistoquímica con proteína S100 +++ CD $99+++$, TLE-1 ++, Ki67 +++, revelando Melan A: negativo, PanK: negativo, CK 7: negativo, CK 20: negativo, con resultado de sarcoma sinovial poco diferenciado con alta actividad proliferativa (Figura 3). Ante este resultado, se programó para linfadenectomía femoral izquierda, la cual reportó 14 ganglios linfáticos, sin evidencia de lesión. El paciente cursó con buena evolución sin tratamiento oncológico.

\section{DISCUSIÓN}

Los sarcomas de tejidos blandos son un grupo de neoplasias mesenquimatosas poco frecuentes, que suponen menos del $1 \%$ de todos los tumores malignos y, únicamente, $2 \%$ de todas las muertes por cáncer. ${ }^{8}$ En el caso de los niños, estos sarcomas representan el $8 \%$ de todas las neoplasias malignas. ${ }^{9}$ El sarcoma sinovial representa entre 5 y $10 \%$ de todos los sarcomas de tejidos blandos y ocupa el cuarto en frecuencia después del histiocitoma fibroso maligno, el liposarcoma y el rabdomiosarcoma. ${ }^{2}$ Existe un pico de incidencia en la tercera década de la vida, pero también puede tener una presentación en adultos mayores de 60 años. ${ }^{10}$

Esta neoplasia es ligeramente más frecuente en hombres que en mujeres, con una relación 1.2:1, y no presenta predilección racial. ${ }^{3}$ Su etiología es desconocida, sin embargo,

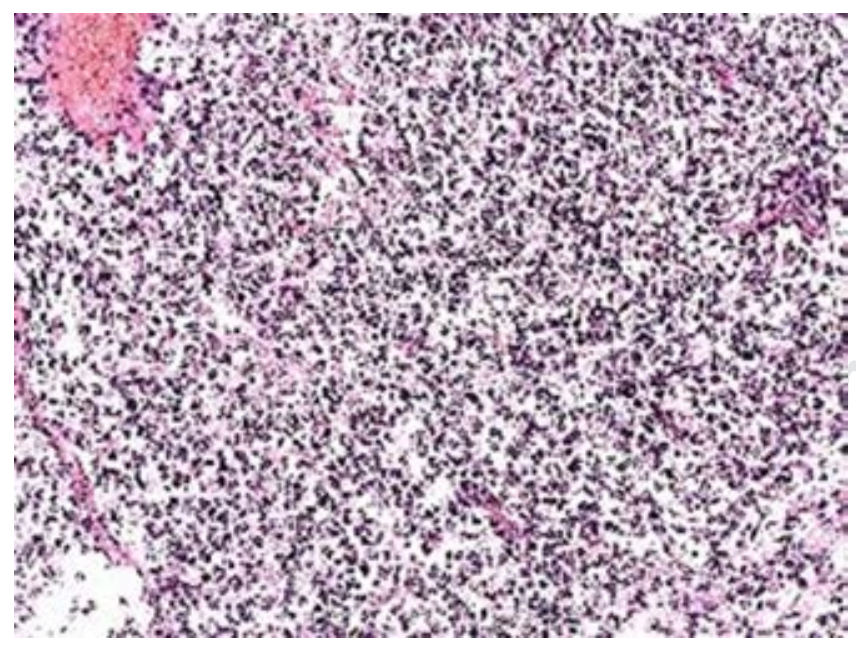

Figura 1: Panorámica (10x) del tumor con hematoxilina eosina.

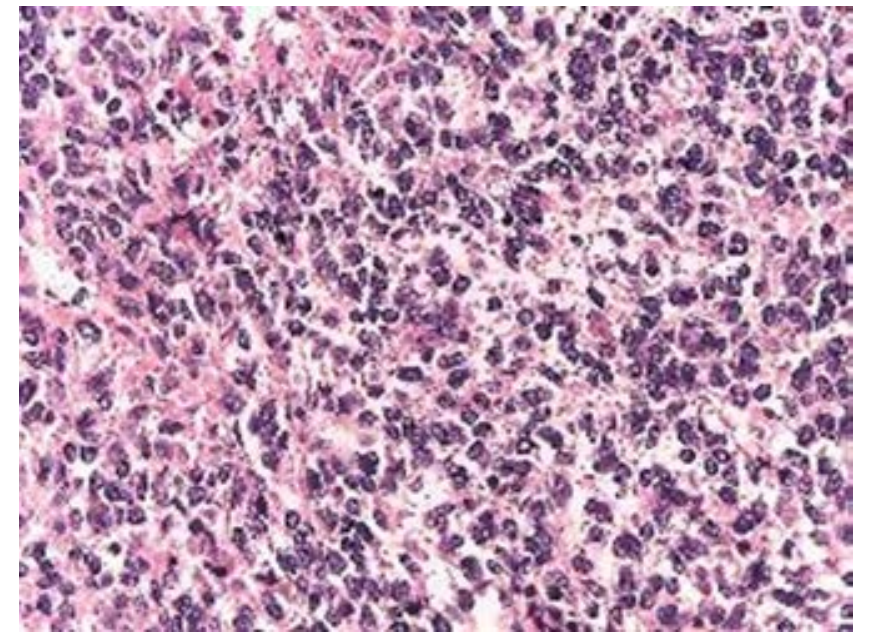

Figura 2: Panorámica (40x) del tumor teñido con hematoxilina eosina, con sospecha de hidradenocarcinoma.

se han identificado diversos factores como son: síndromes genéticos, radioterapia o quimioterapia previa, carcinógenos químicos, linfedema crónica e irritación. ${ }^{8}$ Se localiza fundamentalmente en la rodilla y el tobillo, pero también puede hacerlo cerca de la cadera, hombros y codos. ${ }^{8}$ Un origen distinto a las extremidades representa el 20\%, entre ellos, los sitios más frecuentes son el tórax (19\%), el retroperitoneo $(15 \%)$, la región de la cabeza y cuello $(9 \%)$, y el mediastino. ${ }^{5}$ Otras localizaciones menos frecuentes son intravasculares, intraarticulares, pleuropulmonares, los riñones, la próstata y la glándula tiroides o el pericardio. ${ }^{6}$

Generalmente tienen un tamaño de presentación menor de $5 \mathrm{~cm}$, aunque pueden tener un tamaño de más de 15 cm. ${ }^{7}$ Aproximadamente, el 50\% de los casos presentará metástasis al diagnóstico. La mayoría de los casos son metástasis pulmonares. El 10-12\% las representan las metástasis ganglionares; las metástasis intraabdominales y hepáticas son raras. ${ }^{7}$

Histológicamente, se divide en bifásico, monofásico fibroso, monofásico epitelial y pobremente diferenciado. ${ }^{2}$ El tipo bifásico está constituido por un componente fusiforme mezclado de forma variable con zonas epiteliales, las cuales están compuestas predominantemente por glándulas irregulares y escasas células fusiformes. El tipo monofásico fibroso o fusocelular está constituido por una densa población de células fusiformes en cordones, formado por células pequeñas-medianas que contienen núcleos pálidos, citoplasma disperso y bordes celulares inaparentes; éste suele ser el subtipo más frecuente. ${ }^{1} \mathrm{Si}$ no se llevan a cabo estudios de citogenética, el tipo monofásico epitelial o glandular es prácticamente indistinguible de un adenocarcinoma. ${ }^{11}$ La inmunohistoquímica tiene utilidad diagnóstica y pronóstica. Los marcadores epiteliales son los 
más importantes, entre ellos, se incluyen la panqueratina, el CAM 2.5 y el antígeno de membrana epitelial (EMA). ${ }^{12}$ En el tipo epitelial, además de los mencionados, se encuentran también el antígeno carcinoembrionario, del gen BCL-2 y las citoqueratinas $\mathrm{AE} 1 / \mathrm{AE} 3,7$ y 19 . En el tipo fusocelular, se reconoce la E-caderina, vimentina, desmina, CD99 y CD56. Wong y su equipo describieron que el diagnóstico se debe complementar con la negatividad a CD34. ${ }^{13}$

En más del $90 \%$ de los sarcomas sinoviales, se presenta una translocación característica entre el cromosoma $X$ y $18 t(X ; 18)(p 11 ; q 11) .{ }^{2}$ De acuerdo con Mandujano y colaboradores, dicha translocación genera en dos tercios de los casos la fusión SYT-SSX1, y en un tercio de los casos, la fusión SYT-SSX2; en casos excepcionales, hay fusión SYTSSX4. Se considera que las proteínas de fusión SYT-SSX están involucradas en la represión aberrante de E-caderina, la cual es necesaria para la diferenciación epitelial de dichos tumores. ${ }^{14}$

En todo paciente con sospecha clínica de sarcoma sinovial se debe incluir en su manejo la realización de radiografías simples con el fin de descartar otras etiologías. En 15 a 20\% de ellos existe una reacción perióstica, erosiones superficiales o invasión ósea. La imagen típica es una masa no homogénea, septada, de baja a intermedia intensidad con márgenes infiltrados. ${ }^{9}$ La resonancia magnética es el estudio de imagen de elección para el diagnóstico y para la valoración del tratamiento quirúrgico. Sin embargo, los estudios de imagen son poco específicos y en todos los casos es necesaria la biopsia para confirmar dicho diagnóstico. ${ }^{2}$

El sarcoma sinovial es una neoplasia de mal pronóstico. Se ha descrito una supervivencia a cinco años de 36 a $76 \%$. Son factores de buen pronóstico la edad menor a 15 años,

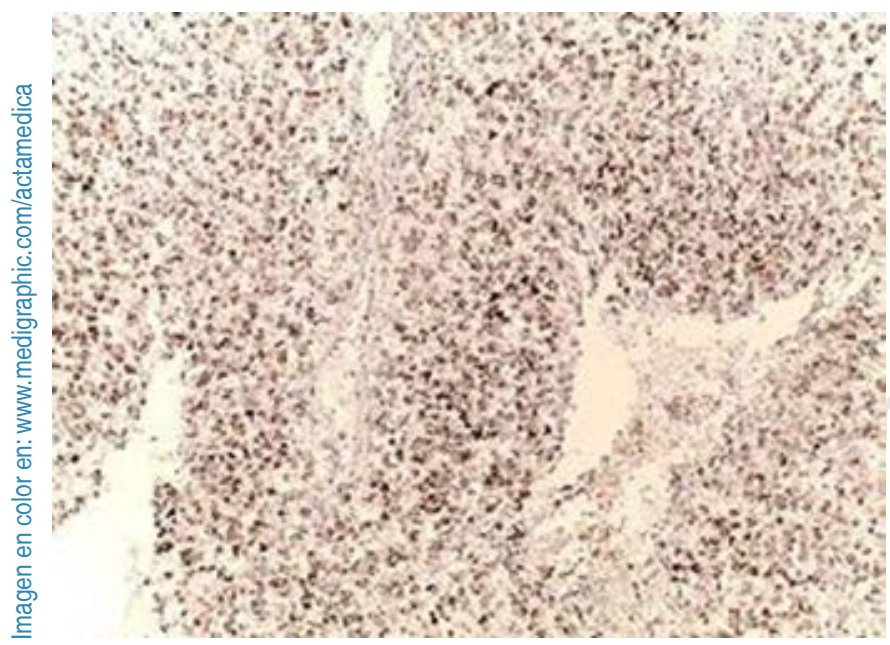

Figura 3: Tumor teñido con inmunohistoquímica para TLE-1, específico para sarcoma sinovial (10x). un tamaño tumoral menor a $5 \mathrm{~cm}$ y una localización distal en las extremidades. ${ }^{2}$ Los tumores altamente calcificados también presentan un mejor pronóstico, con una supervivencia a cinco años de hasta $80 \%$. Los factores de mal pronóstico son: tamaño tumoral mayor de $5 \mathrm{~cm}$, afectación profunda, resección quirúrgica inadecuada, edad mayor de 20 años, subtipo monofásico, localización en tronco y abdomen, y actividad mitótica mayor de 10 por campo. ${ }^{10}$ La estadificación del sarcoma sinovial se realiza con base en el sistema clásico de estadificación tumoral modificada por la Comisión Americana del Cáncer, con la inclusión de factores clínicos (tamaño, localización, presencia o ausencia de enfermedad metastásica) y criterios histológicos de la biopsia (número de mitosis, atipias y necrosis). ${ }^{2}$

La base del tratamiento del sarcoma sinovial es la resección quirúrgica completa con márgenes quirúrgicos óptimos. Las recidivas locales igualmente tienen un tratamiento quirúrgico. ${ }^{6}$ La quimioterapia con doxorrubicina e ifosfamida ha mostrado eficacia en el tratamiento, sobre todo para disminuir el tamaño tumoral y facilitar la cirugía; también se puede utilizar en pacientes con tumores de alto grado, alto riesgo de recurrencia local o enfermedad metastásica. ${ }^{13}$

En el caso de los tumores irresecables, aquéllos con tamaño mayor a $5 \mathrm{~cm}$, la radioterapia es el tratamiento de elección. Se puede administrar de forma externa, como braquiterapia o mediante radioterapia de intensidad modulada. En los casos de sarcoma sinovial en edad pediátrica, su uso es controvertido. ${ }^{3}$

El sarcoma sinovial es un tumor infrecuente, el cual se debe sospechar en cualquier masa de tejidos blandos con localización periarticular, incluso cuando puede presentarse en cualquier parte del cuerpo. En el caso presentado, el primer diagnóstico de sospecha fue hidradenocarcinoma, para posteriormente realizar los estudios pertinentes. Sin embargo, el resultado de los marcadores de inmunohistoquímica arrojó diagnóstico de sarcoma sinovial de los tejidos blandos debido a la negatividad a los marcadores de queratina Pank, CK7, CK 20, los cuales descartaron la posibilidad de hidradenocarcinoma. Los sarcomas sinoviales con diferenciación epitelioide pueden asemejar carcinomas de glándulas sudoríparas, la positividad a proteína s100 podría orientar a melanoma amelánico o un tumor de vaina nerviosa, pero la positividad mínima a HMB 45 y Melan A no apoyan el diagnóstico.

\section{CONCLUSIONES}

La importancia de este artículo es reportar un caso de sarcoma sinovial de localización en extremidad inferior y los hallazgos inmunohistoquímicos correspondientes con la presentación común de esta neoplasia. 


\section{REFERENCIAS}

1. Vlenterie M, Jones RL, Van der Graaf WT. Synovial sarcoma diagnosis and management in the era of targeted therapies. Curr Opin Oncol. 2015; 27 (4): 316-322.

2. Bravo-Pérez M, López-López S, García-Porrúa C. Sarcomas sinoviales. Seminarios de la Fundación Española de Reumatología. 2009; 10 (1): 29-34.

3. Song S, Park J, Kim HJ, Kim IH, Han I, Kim HS et al. Effects of adjuvant radiotherapy in patients with synovial sarcoma. Am J Clin Oncol. 2017; 40 (3): 306-311.

4. Mitsuhashi A, Nagai Y, Suzuka K, Yamazawa K, Nojima T, Nikaido $T$ et al. Primary synovial sarcoma in fallopian tube: case report and literature review. Int J Gynecol Pathol. 2007; 26 (1): 34-37.

5. Zanardi E, Maruzzo M, Montesco MC, Roma A, Rastrelli M, Basso U. Response to trabectedin in a patient with advanced synovial sarcoma with lung metastases. Anticancer Drugs. 2014; 25 (10): 1227-1230.

6. Flores-Aguilar S R, Lecuona-Huet N, Córdova-Quintal P, LacayValenzuela M E, Cárdenas-Sánchez AM. Sarcoma sinovial monofásico epiteloide en extremidad torácica, como diagnóstico diferencial de pseudoaneurisma trombosado: reporte de un caso del Hospital General de México "Dr. Eduardo Liceaga". Revista Mexicana de Angiología. 2017; 44 (2): 77-80.

7. Terra S B, Aesif SW, Maleszewski JJ, Folpe AL, Boland, JM. Mediastinal synovial sarcoma: Clinicopathologic analysis of 21 cases with molecular confirmation. Am J Surg Pathol. 2018; 42 (6): 761-766.
8. Fisher C, De Bruijn DRH, Geurts van Kessel A. Synovial Sarcoma. World Health Organization Classification of Tumours: pathology and genetics of soft tissue and bone. IARC, Lyon. 2002: 200-204.

9. Chotel F, Unnithan A, Chandrasekar CR, Parot R, Jeys L, Grimer RJ. Variability in the presentation of synovial sarcoma in children: a plea for greater awareness. J Bone Joint Surg Br. 2008; 90 (8): 1090-1096.

10. Kerouanton A, Jimenez I, Cellier C, Laurence V, Helfre S, Pannier S et al. Synovial sarcoma in children and adolescents. Journal Of Pediatric Hematology/Oncology. 2014; 36 (4): 257-262.

11. West RB. Expression profiling in soft tissue sarcomas with emphasis on synovial sarcoma, gastrointestinal stromal tumor, and leiomyosarcoma. Advances in Anatomic Pathology. 2010; 17 (5): 366-373.

12. Chrisinger JS, Salem UI, Kindblom LG, Amini B, Hansson M, Meis JM. Synovial sarcoma of peripheral nerves. Am J Surg Pathol. 2017; 41 (8): 1087-1096.

13. Wong CS, Harris A, Kennedy R, Houghton OP, Carey PD. A rare case of retroperitoneal synovial sarcoma. JRSM. 2018; 9 (4): 1-4.

14. Mandujano-Álvarez GJ, Ortiz-Hidalgo C. Sarcoma sinovial. Estudio clínico-patológico e inmunohistoquímico de 21 casos con énfasis en el TLE-1, p16 y ciclina D1. Patología Rev Latinoam. 2011; 49 (1): $1-10$.

Conflicto de interés: Los autores de este artículo declaran que no existe ningún tipo de conflicto de intereses. 